\title{
AÇÕES EDUCACIONAIS NA ESTRATÉGIA SAÚDE DA FAMÍLIA: PERSPECTIVAS DOS PROFISSIONAIS DE ENFERMAGEM
}

\section{EDUCATIONAL ACTIONS IN THE FAMILY HEALTH STRATEGY: PERSPECTIVES OF NURSING PROFESSIONALS}

\author{
Rayane Moreira de Alencar'; Pedro Paulo Rodrigues II*; Alessandra Bezerra de Brito III; Magaly Lima MotalV
}

\begin{abstract}
Resumo. A educação em saúde na Estratégia Saúde da Família (ESF) é um procedimento voltado para reorganização dos serviços de saúde, configurando-se como sendo uma ferramenta potente para identificar e solucionar as fragilidades que ainda permeiam as ações de educação em saúde pelos profissionais da ESF. Dessa forma, esse estudo teve como objetivo identificar a concepção dos profissionais de enfermagem da Estratégia Saúde da Família sobre as ações educacionais. Tratouse de um estudo descritivo e exploratório, com abordagem qualitativa, realizado com uma amostragem de sete enfermeiros e nove técnicos em enfermagem, os quais eram profissionais de enfermagem de nove unidades da Estratégia Saúde da Família da zona urbana do município de Exu no interior do estado do Pernambuco. A coleta de dados foi feita por meio de entrevista semiestruturada, com roteiro pré-elaborado, contendo questões subjetivas, desenvolvida de dezembro de 2016 a fevereiro de 2017. Os dados foram analisados conforme a Técnica de Análise de conteúdo na modalidade Temática. A partir da análise de conteúdo emergiram três categorias: Concepções acerca da Educação Associada à Saúde; Desenvolvimento de Ações Educativas e Desafios Encontrados na Efetivação de Ações Educativas na Atenção Primária. Conclui-se que as ações educativas ainda são apontadas como sendo uma prática profissional voltada para o caráter preventivo, as quais acontecem através de metodologias que reduzem a distância entre o conhecimento dos profissionais de enfermagem e da comunidade, bem como a demanda excessiva e consequentemente a falta de tempo disponível como principal obstáculo para efetivação da educação em saúde.
\end{abstract}

PALAVRAS-CHAVE: Atenção Primária à Saúde. Educação em Saúde. Enfermagem em Saúde Pública.

Abstract. Health education in the Family Health Strategy (FHS) is a procedure aimed at reorganizing health services, working as a powerful tool to identify and solve weaknesses that still permeate health education actions by FHS professionals. Thus, this study aimed to identify nursing professionals' educational action concepts in the Family Health Strategy. It was a descriptive and exploratory study, with a qualitative approach, carried out with a sample of seven nurses and nine nursing technicians, who were professionals from nine units of the Family Health Strategy in the urban area of the city of Exu in the countryside of the state of Pernambuco. Data collection was carried out through a semi-structured interview with a pre-prepared script containing subjective questions developed from December 2016 to February 2017. Data were analyzed according to the Content Analysis Technique in the Thematic mode. From the content analysis, three categories emerged: Conceptions about health associated education; Development of educational actions; and Challenges found in carrying out educational actions in primary care. It is concluded that educational actions are still identified as a professional practice focused on preventive care, which take place through methodologies that reduce the gap between the knowledge of nursing professionals and the community, as well as the excessive demand and consequently the lack of available time as the main obstacle to the realization of health education.

KEYWORDS: Primary Health Care. Health Education. Public Health Nursing. 


\section{INTRODUÇÃO}

O Programa Saúde da Família (PSF) foi criado pelo Ministério da Saúde, em 1994, no intuito de fortalecer as conexões com a população e em busca de aproximar os profissionais da comunidade, visando um maior desempenho e zelo aos usuários. Atualmente, é nomeado como Estratégia Saúde da Família (ESF), constituído por equipes que almejam estruturar os sistemas municipais de saúde pública e possuem o propósito de reorganizar o modelo de atenção no Sistema Único de Saúde (SUS). ${ }^{1}$

No âmbito da ESF as equipes multiprofissionais atuam com públicos e programas distintos, abrangendo inúmeras atribuições. Dentre as pertinências da equipe, estão as atuações educativas, tornandoos agentes de transformações, sejam elas com enfoque individual, ou coletivamente, no contexto biopsicossocial de atenção à família, conferindo ao profissional a função de ser o facilitador do processo de educação em saúde. ${ }^{2}$

A educação em saúde é vista como uma medida utilizada na Atenção Primária à Saúde (APS), para concretizar a aproximação entre os serviços de saúde e a comunidade, entre o educador e o educando, na dinâmica de ensino-aprendizagem. Tratase de um processo educativo complexo, em permanente construção, que se dá por meio da troca de saberes/experiências, objetivando debater e promover a tomada de decisão a respeito das ações de saúde. ${ }^{3}$

As equipes da ESF são compostas por profissionais distintos, dentre eles a equipe de enfermagem que desenvolve cuidados essenciais neste nível de assistência. O enfermeiro, junto com o técnico em enfermagem, é constantemente associado ao papel de cuidador e, ao realizar a assistência à saúde, educa e estimula a corresponsabilização com os demais, valorizando a autonomia do sujeito sobre sua saúde. Assim sendo, a educação em saúde pode ser avaliada como uma maneira de cuidar e o cuidado um modo de educar. 4 As ações de educação em saúde devem ser desenvolvidas por todos os profissionais da saúde, através da criação de uma relação dialógico-reflexiva entre os profissionais e as pessoas, com o propósito de conscientizar todos a respeito da sua saúde e percepção como participante ativo na transformação de vida. 5

A educação em saúde éuma estratégia voltada para reorganização dos serviços à saúde na APS, com destaque na prevenção de doenças e na promoção à saúde e, configurando-se como sendo uma ferramenta potente para identificar e solucionar as fragilidades que ainda permeiam as ações de educação em saúde pelos profissionais da ESF. ${ }^{6}$ A atuação da enfermagem na inovação das estratégias de educação em saúde ainda é envolvida de dificuldades porque se acredita que a enfermagem deva criar e/ou utilizar metodologias ativas, estimulando para que sejam empregadas de maneira organizada e sistematizada, através de pesquisas científicas, estimulando, assim, essa discussão. ${ }^{7}$

$\mathrm{Na}$ ESF, as ações educativas implicam em um olhar diferenciado dos profissionais sobre o conceito de saúde, tendo a integralidade como princípio fundamental, evidenciando a relevância desta temática. ${ }^{8}$ Nessa perspectiva, esse estudo possui como objetivo identificar a concepção dos profissionais de enfermagem da Estratégia Saúde da Família sobre as ações educacionais. 


\section{MATERIAL E MÉTODOS}

Estudo descritivo e exploratório, com abordagem qualitativa, desenvolvido de dezembro de 2016 a fevereiro de 2017, nas nove ESF da zona urbana do município de Exu no interior do estado do Pernambuco. Incluíram-se todos os enfermeiros e técnicos em enfermagem atuantes nesses dispositivos, considerando a necessidade de vivências na unidade. Excluíram-se aqueles que estavam de férias, licença, ou qualquer tipo de afastamento durante $o$ período de coleta de dados, bem como, os que não possuíam disponibilidade para participar da pesquisa. Dos 18 profissionais abordados para participação da pesquisa, houve indisponibilidade de dois enfermeiros, resultando em uma amostra final de sete enfermeiros e nove técnicos em enfermagem.

Os dados foram obtidos por meio de entrevista semiestruturada com roteiro pré-elaborado contendo questões subjetivas que possibilitassem atingir os objetivos

\section{RESULTADOS}

A partir da análise de conteúdo das falas dos sujeitos da pesquisa emergiram três categorias: "Concepções acerca da educação associada à saúde", "Desenvolvimento de ações educativas" e "Desafios encontrados na efetivação de ações educativas na atenção primária".

\section{Concepções acerca da educação associada à saúde}

Quando questionados sobre o entendimento que eles possuíam no que propostos. As entrevistas ocorreram na unidade de saúde onde os profissionais exercem suas atividades laborais, em sala reservada, durante os dias e horários de funcionamento.

Todos os dados foram analisados conforme a Técnica de Análise de conteúdo na modalidade Temática, ${ }^{9}$ que é uma das estratégias usadas para a pesquisa qualitativa com grande rigor e cientificidade.

Para apresentação dos resultados e garantia do anonimato dos sujeitos da pesquisa, esses foram identificados através de uma letra seguida de um número.

Escolheu-se a letra $E$ para caracterizar os enfermeiros e $T$ para os técnicos em enfermagem ( $E_{1}, E_{2} \ldots E_{7}$; T1, T2,... T9). O estudo foi aprovado sob o CAAE 54458215.1.0000.5048 e todos os participantes incluídos na pesquisa assinaram o Termo de Consentimento Livre e Esclarecido, formalizando suas participações. diz respeito às ações educacionais na ESF, os enfermeiros e técnicos em enfermagem destacaram a importância do diálogo com os pacientes e das orientações para manutenção de uma vida saudável.

Educação em saúde é quando associamos os nossos cuidados a práticas cotidianas que melhoram a qualidade de vida do paciente. A gente conversa com eles sobre maneiras de manter-se saudável, prevenção de riscos, a importância de atividade 
física, estas coisas no geral. (E1)

É quando o profissional durante o atendimento fala sobre prevenção, sobre os cuidados que o paciente deve ter, quando ele não fala só da doença e sim sobre outros aspectos positivos. (E2)

É quando a gente faz campanha, vai as casas e orienta as pessoas. Eu não entendo muito disso, mas acho que é assim. (T8)

Não sei te dizer bem, mas eu acredito que seja quando a gente conversa sobre o paciente e diz maneiras de prevenir as doenças, quando fala de alimentação, faz palestras [...] (T2)

\section{Desenvolvimento de ações educativas}

A palestra foi reconhecida como a principal prática educativa trabalhada na ESF. Através das falas dos participantes, constatase o uso de metodologias tradicionais, voltadas para um processo de diálogo vertical sem participação ativa da coletividade.

A gente costuma fazer palestra com a população, aproveitar dias que não tem tantos atendimentos agendados. $O$ NASF também costuma vir e conversar com os pacientes, isso ajuda muito, porque eles fazem isso enquanto a gente vai atendendo. (E6)

Se for o que eu estou pensando a gente faz sim. Distribui alguns panfletos, faz campanha, estas coisas né, isso quando dá tempo. (T7)

Eu aproveito o momento em que o paciente está no consultório, converso com ele sobre o seu quadro, dou orientações e peço para que ele me diga se tem alguma dúvida, tento sempre priorizar os grupos de risco para trabalhar a educação em saúde [...] as palestras são sempre boas para isso. (E4)

\section{Desafios encontrados na efetivação de ações educativas na atenção primária}

No que se refere aos principais obstáculos enfrentados no processo de efetivação da educação em saúde na unidade, os sujeitos da pesquisa apontaram a demanda excessiva e, consequentemente, a falta de tempo disponível para essas ações como os desafios mais presentes, conforme explícito nas falas que seguem:

Aqui é sempre cheio. Tem dias que não temos tempo nem de atender todo mundo, você sabe como é PSF, então a gente acaba priorizando a consulta em si. Acho que o principal problema é que temos que escolher o que dá pra fazer, porque são tantos papéis pra preencher que não dá para ter tempo livre. (E3)

[...] a gente não tem tempo para fazer muito isso porque aqui está sempre cheio, acho que a demanda excessiva é o principal obstáculo. (T4)

Ninguém ajuda [...] ninguém tem a iniciativa de dizer como é que a gente tem que fazer ou de dar material para a gente trabalhar, além da falta de tempo [...]. (T1)

[...] não temos um espaço ideal para trabalhar educação em saúde e muitas vezes a própria população não está interessada [...] porque nem eles, nem nós, temos tempo. (E5) 


\section{DISCUSSÃO}

A partir do estudo e das categorias que emergiram, percebe-se que o discurso remete prioritariamente às medidas de prevenção. Constata-se que alguns dos participantes não realizaram a associação direta as práticas à promoção da saúde, corroborando com os resultados encontrados com um estudo feito com profissionais das ESF do município de Santa Marina, no Paraná, com a finalidade de avaliar suas compreensões a respeito da educação em saúde. ${ }^{10}$

As concepções acerca da educação associada à saúde devem se consolidar como um processo educativo mais próximo da reflexão, do diálogo e da problematização. ${ }^{11} \mathrm{O}$ modelo educativo dialogal e problematizador são avaliados como sendo o ideal para a área da saúde, de maneira especial pela possibilidade de aproximar a população assistida pela ESF aos serviços de saúde, assim como uma medida para despertá-lo no protagonismo dos usuários na melhoria de suas condições de vida. ${ }^{12}$

A educação em saúde ainda encontra-se intimamente ligada a busca da eliminação da doença, porém que as equipes da ESF devem atuar considerando as políticas públicas, ambientes apropriados e reorientação dos serviços de saúde para além dos tratamentos clínicos e curativos, bem como, as medidas pedagógicas libertadoras, afetadas com o incremento da solidariedade e da cidadania, dirigindose para as ações que possuem essência no progresso da qualidade de vida e na promoção da saúde. ${ }^{13}$ Educação em saúde não deve limitar-se aos processos patológicos, a mesma deve assegurar o controle dos riscos, a adoção de um estilo de vida saudável e busca por melhores condições de saúde, tornando-a uma metodologia para aperfeiçoar as condições atuais.

A partir dessas preposições a educação deve ocorrer de maneira aberta, horizontal e libertadora, ${ }^{14}$ no qual o educador deve estar sempre disponível para ouvir o que a comunidade tem para relatar e não somente falar, cabendo aos profissionais de enfermagem proporcionar espaços e momentos propícios para construção de diálogos.

No processo de desenvolvimento das ações educativas a categoria sinaliza para a utilização de um diálogo vertical, em que o modelo de comunicação se volta para a transmissão de saberes, provendo atividades educativas por meio do repasse de entendimentos ao outro. O profissional de enfermagem nas ações de educação em saúde assume o papel de ser aquele que sabe e que deve dizer algo, ao mesmo tempo em que o usuário passa a não possuir conhecimentos, devendo recebêlos passivamente. ${ }^{15-16}$ Quando se realiza ações educativas como um processo de depósito de ensinamentos, considera-se o usuário como uma caixa vazia.

Deve-se dar ênfase ao diálogo e a troca de saberes em ações que vão além da realização de palestras, realizando atividades interligadas com - lazer e a interação social, a exemplo de festas e eventos culturais, bingos, mobilizações sociais, formação de grupos de convivência, oficinas de arte, música, dança e outras ações ligadas à cultura popular. ${ }^{17}$ É necessário desenvolver ações que possam potencializar as medidas de educação popular em saúde, alterando o entendimento além das muralhas do serviço de saúde. 
As ações educativas em saúde na ESF devem ser realizadas por todos os profissionais da área com o propósito de construir, em conjunto com a comunidade, vínculo e compromisso, procurando melhorias nas condições de vida, por meio do envolvimento de todos. ${ }^{18}$ A utilização dessas ações como método na assistência na enfermagem transcende os preceitos básicos do cuidado, pois é através da educação que o enfermeiro irá potencializar a sua habilidade de cuidar, ${ }^{19}$ promovendo assim a inclusão social e a promoção da autonomia das populações na participação em saúde. ${ }^{20-21}$

Em um estudo realizado para verificar a importância do enfermeiro nas ações de educação na ESF, evidenciouse que esses profissionais não realizavam atividades de educação em saúde pela falta de tempo para desenvolver atividades de promoção e prevenção à saúde, visto que estavam atarefados com a prática assistencial e com a função de gestor da ESF. 22-23

Predominam atividades educativas voltadas para o público-alvo de uma área profissional específica, aspecto que

\section{CONCLUSÃO}

As ações educativas ainda são apontadas como sendo uma prática profissional voltada para o caráter preventivo, dando-se por meio de metodologias que acentuam a distância entre os saberes dos profissionais e da comunidade, direcionando-se a grupos prioritários. As dificuldades encontradas envolvem questões de formação, gerenciais, institucionais e relativas aos processos de trabalho.

Como contribuições para a saúde, acredita-se que as ações educativas não caracteriza a fragmentação das ações de saúde e a prevalência do trabalho individualizado por categorias, no modo a organizar o processo de trabalho em saúde, deixando de lado a interdisciplinaridade e o trabalho multiprofissional. ${ }^{24}$

Estudos vêm sendo desenvolvidos com o propósito de localizar o desenvolvimento de ações educativas pelos profissionais de saúde que atuam junto à comunidade, constatando-se que a maioria dessas não é empregada com frequência, devido à desorganização da demanda, à baixa escolaridade dos usuários e à resistência da população às ações educativas, corroborando com o estudo aqui discutido. ${ }^{25}$

Para que a educação em saúde se torne uma atividade no cotidiano do funcionamento de ESF, se faz necessário o entendimento das finalidades da estratégia por todos os membros da equipe e, sobretudo pela comunidade. A ausência de recursos humanos tem dificultado a realização do trabalho, em especial a parte de educação em saúde, pois a maior parte direciona ao atendimento da demanda. ${ }^{26-27}$

devem ser executadas apenas na existência de tempo ocioso, priorizando as de cunho assistencial, o profissional de enfermagem deve considerar a importância de ambas no cuidado integral a comunidade. A educação continuada constitui-se como uma ferramenta para a qualificação e capacitação dos profissionais, possibilitando mecanismos para que eles possam entender e corresponder às necessidades de saúde da população.

Quanto às implicações, a pesquisa 
estimula reflexões a respeito do impacto da sobrecarga de atividades a serem desenvolvidas noâmbito da ESF pela equipe de enfermagem, abordando aspectos gerenciais

\section{REFERÊNCIAS BIBLIOGRÁFICAS}

1. Brasil. Ministério da Saúde. Atenção Básica e a Saúde da Família. Brasília: Ministério da Saúde, 2004. Disponível em: <http://dab.saude.gov.br/portaldab/ ape_esf.php >. Acesso em: 26 set. 2016.

2. Barbiani R, Nora CRD, Schaefer R. Práticas do enfermeiro no contexto da atenção básica: scoping review. Nursing practices in the primary health care context: a scoping review. Rev Latino-Am Enfermagem. 2016; 24:e2721. Doi: http://dx.doi. org/10.1590/1518-8345.0880.2721

3. Junqueira MAB, Santos FCS. A educação em saúde na Estratégia Saúde da Família sob a perspectiva do enfermeiro: uma revisão de literatura. Rev Ed Popular, Uberlândia, 2013 Jan/June; [cited 2016 Sept 26]; 12(1): 66-80. Doi: http://dx.doi.org/10.14393/ REPv12n12013-arto6.

4. Galavote HS, Zandonada E, Garcia ACP, Freitas PSS, Seidl H, Contarao PC, et al. The nurse's work in primary health care Trabajo del enfermero en la atención primaria de salud. Esc Anna Nery. 2016 Jan/ Mar; 20(1): 90-98. Doi: 10.5935/1414-8145.20160013

5. Mallmann DG, Galindo Neto NM, Sousa JC, Vasconcelos. Health education as the main alternative to promote the health of the elderly. Ciênc saúde coletiva, Rio de Janeiro. 2015 June; 20(6):1763-72. Doi: http://dx.doi.org/10.1590/1413-81232015206.02382014

6. Santili PGJ, Tonhom SFR, Marin MJS. Health education: challenges for implementation. Investigación Cualitativa en Salud. 2016 June; 2(3): 1147-56. Available from: https://proceedings.ciaiq.org/ index.php/ciaiq2016/article/view/867

7. Rodrigues CCFM, Carvallho DPSRP, Salvador PTCO, Medeiros SM, Menezes RMP, Ferreira Júnior MAF, et que se sobrepõem as possibilidades de uma atuação que fortalece a promoção da saúde e supera um modelo biomédico.

al. Innovative nursing education from the perspective of epistemologies of the South. Esc Anna Nery, Rio de Janeiro. 2016 Apr/June; 20(2): 384-89. Doi: http:// dx.doi.org/10.5935/1414-8145.20160053

8. Bomfim ES, Araújo IB, Oliveira BG, Moreira RM, Rocha RM, Boery RNSO. Educação permanente em saúde: discussão das práticas educativas na estrategia de saúde da familia. Rev Enferm Ufpe, Recife. 2016 Aug; 10(8): 2833-37. Available from: http://www.revista.ufpe.br/revistaenfermagem/ index.php/revista/article/view/8988/pdf_10746.

9. Bardin L. Análise de conteúdo. São Paulo: Edições 70; 2011.

10. Roecker S, Nunes EFPA, Marcon SS. O trabalho educativo do enfermeiro na Estratégia Saúde da Família. Texto Contexto Enferm, Florianópolis, 2013 Jan/Mar; 22(1): 157-65. Doi: http://dx.doi.org/10.1590/ S0104-07072013000100019.

11. Simon E, Jezine E, Vasconcelos EM, Ribeiro KSQS. Metodologias ativas de ensino-aprendizagem e educação popular: encontros e desencontros no contexto da formação dos profissionais de saúde. Interface, Botucatu. 2014; 18 Supl 2: 1355-64. Doi: http://dx.doi.org/10.1590/1807-57622013.0477.

12. Arantes $\sqcup$, Shimizu HE, Merchán-Hamann E. Contribuições e desafios da Estratégia Saúde da Família na Atenção Primária à Saúde no Brasil: revisão da literatura. Ciênc saúde coletiva, Rio de Janeiro. 2016; 21(5): 1499-09. Doi: 10.1590/141381232015215.19602015 .

13. Leite AGA, Sousa JCM, Feitosa ANA, Vieira AG, Quental OB, Assis EV. Health education practices in the family health strategy: an integrative review 
of literature. Rev enferm UFPE, Recife. 2015 Dec; 9(Supl. 10): 1572-79. Doi: 10.5205/reuol.8463-73861-2SM.0910sup201525.

14. Almeida ER, outinho $C B$, Leite MTS. Prática pedagógica de enfermeiros de Saúde da Família no desenvolvimento da Educação em Saúde. Interface, Botucatu. 2016 Feb; 20(57): 389-402. Doi: http:// dx.doi.org/10.1590/1807-57622015.0128.

15. Saito DYT, Zoboli ELCP, Schveitzer MC, Maeda ST. Usuário, cliente ou paciente? Qual o termo mais utilizado pelos estudantes de enfermagem? Texto Contexto Enferm, Florianópolis, 2013 Jan-Mar; 22(1): 175-83. Doi: http://dx.doi.org/10.1590/S010407072013000100021.

16. Freire P. Pedagogia da autonomia: saberes necessários à prática educativa. São Paulo: Paz e Terra, 1996.

17. Xavier PRH, Lopes SMB. As práticas do Assistente Social e sua articulação com a Equipe de Saúde de um Hospital de Curitiba. Serv Soc Rev, Londrina. 2016 Jan/June; 18(2): 132-50. Doi:10.5433/1679-4842.2016v1 8n2p132.

18. Roecker S, Nunes EFPA, Marcon SS. O trabalho educativo do enfermeiro na Estratégia Saúde da Família. Texto Contexto Enferm, Florianópolis, 2013
Jan/Mar; 22(1): 157-65. Doi: http://dx.doi.org/10.1590/ S0104-07072013000100019.

19. Rosa J, Faccin C, Delegrave D, Argenta C, Fransciscatto LHG. Ações educativas de assistência em enfermagem em ambiente hospitalar: a atenção a pais e familiares de neonatos em fototerapia. Rev Enferm FW. 2013; 8(8): 154-65. Available from: revistas.fw.uri.br/index.php/revistadeenfermagem/ article/download/483/881.

20. Pereira FGF, Caetano JA, Moreira JF, Ataíde $M B C$. Práticas educativas em saúde na formação de acadêmicos de enfermagem. Cogitare Enferm. 2015 Apr/June; 20(2): 332-37. Doi: http://dx.doi. org/10.5380/ce.v20i2.39767.

21. Cavalcanti PCS, Oliveira Neto AV, Sousa MF. Quais são os desafios para a qualificação da Atenção Básica na visão dos gestores municipais?. Rev Saud debat. 2015 Apr/June; 39(105): 323-36. Doi: http://dx.doi. org/10.1590/0103-110420151050002323.

22. Costa GM, Figueredo RC, Ribeiro MS. A importância do enfermeiro junto ao PSE nas ações de educação em saúde em uma escola municipal de Gurupi - TO. RcITPA, Araguaína. 2013 Apr; 6(2). Disponível em: <https://assets.itpac.br/arquivos/ Revista/62/6.pdf 